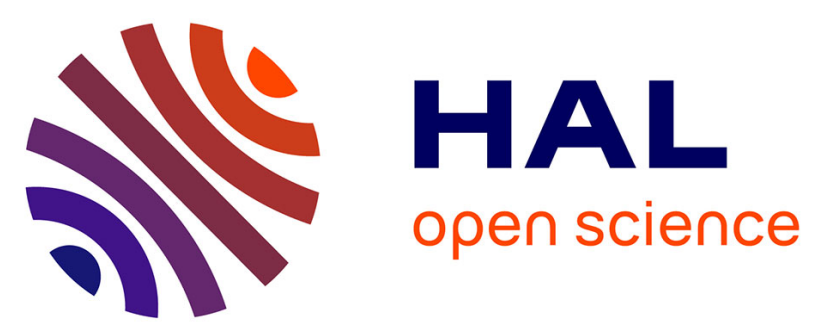

\title{
Maternal antibiotic treatment peripartum has differential consequences on protein expression of intestinal cytoprotective heat shock proteins in her offspring
}

\author{
Marie-Edith Arnal, Jean Paul Lalles
}

\section{To cite this version:}

Marie-Edith Arnal, Jean Paul Lalles. Maternal antibiotic treatment peripartum has differential consequences on protein expression of intestinal cytoprotective heat shock proteins in her offspring. Digestive disease week, May 2013, Orlando, United States. Gastroenterology, 144 (5) (Suppl.1), 2013, Gastroenterology. 10.1016/S0016-5085(13)63374-0 . hal-01594349

\author{
HAL Id: hal-01594349 \\ https://hal.science/hal-01594349
}

Submitted on 3 Jun 2020

HAL is a multi-disciplinary open access archive for the deposit and dissemination of scientific research documents, whether they are published or not. The documents may come from teaching and research institutions in France or abroad, or from public or private research centers.
L'archive ouverte pluridisciplinaire HAL, est destinée au dépôt et à la diffusion de documents scientifiques de niveau recherche, publiés ou non, émanant des établissements d'enseignement et de recherche français ou étrangers, des laboratoires publics ou privés.

\section{()(1)(2)}

Distributed under a Creative Commons Attribution - ShareAlikel 4.0 International 
cell type or differentiation. Significant cell loss occurred by 14 hours post infection due to bystander cell apoptosis which was driven by paracrine signaling. Cleavage of cytokeratin 18 demonstrated apoptosis by 4 hours post infection, but the mechanism mediating this cleavage remains unknown as there was no activation of caspase-3. Inhibition of all caspase activity was able to prevent the early drop in TER, but not the later and more significant decline CONCLUSIONS: C parvum will infect IPEC-J2 cells and complete asexual reproduction, but the infection is limited to a portion of the monolayer. Moreover, the apoptotic signaling in C. parvum infected IPEC-J2 cells is distinctly different than that of in vivo infection. These differences in signaling offer an opportunity to isolate the effect of subepithelial factors in C. parvum infection by comparing the IPEC-J2 cellular response to that of the piglet model.

\section{Tu2014}

Expression of EPEC MAP Is Significantly Different Than That of Other Type 3 Secreted Effectors In Vivo

Mai T. Nguyen, Gail A. Hecht

We previously reported the use of a bacterial luciferase expression plasmid containing the $P$. luminescens luxCDABE operon to study the colonization pattern of enteropathogenic Escherichia coli (EPEC) in a murine model. However, the expression patterns of different EPEC effector proteins have not been investigated. EPEC produces 26 core virulence effector proteins, including locus of enterocyte effacement (LEE) and non-LEE proteins. EspF and Map are multifunctional and extensively studied LEE effector proteins while the non-LEE proteins $\mathrm{NleHl}$ and 2 possess significant anti-inflammatory activity. The aim of this study is to investigate the expression patterns of EspF, Map and $\mathrm{NleHl} / 2$ in living mice in order to better illuminate their roles in EPEC pathogenesis in vivo. We hypothesize that the expression of EPEC effector proteins is differentially regulated over the course of infection. Promoters of these effectors were cloned upstream of the luxCDABE operon of pCM10 plasmid, transformed into EPEC, introduced into mice via oral gavage, and luciferase expression under the control of each promoter was measured by bioluminescent using an in vivo imaging system. Bioluminescent images of living mice, excised whole intestines and those longitudinally opened and washed were assessed at $30 \mathrm{~m}, 3 \mathrm{~h}, 6 \mathrm{~h}, 1 \mathrm{~d}, 2 \mathrm{~d}$ and $4 \mathrm{~d}$ post infection (PI). There was no significant difference in the expression of these effector proteins within the first $24 \mathrm{~h}$ with peak expression occurring at $3 \mathrm{~h}$ PI $\left(\sim 10^{7}\right.$ photon/s). Analysis of excised intestines revealed the majority of bioluminescent bacteria localized in the cecum at $3 \mathrm{~h}$ and $24 \mathrm{~h}$, indicating that the cecum is not only the main colonization site of EPEC, but also of EPEC effector protein expression in mice. Interestingly, Map expression was significantly suppressed at $2 \mathrm{~d}$ PI (only 1 of 4 mice expressed detectable signal, $\sim 10^{6}$ photon/ s), and at 4d PI no mice showed a detectable signal. In contrast, EspF, NleHl and $\mathrm{NleH} 2$ were abundantly expressed $\left(10^{5}-10^{7}\right.$ photon/s) over the course of infection. This pattern of Map expression was confirmed in mice exposed to ketamine, an anesthetic drug that prolongs bacterial colonization for at least 30d. To determine if Map expression was indeed regulated by this promoter region, the promoter was truncated to 125 nucleotides and expression determined. Map expression was abolished at all time points suggesting that this effector is under strict promoter regulation with at least one upstream enhancer involved. Interestingly, when pCMIO carrying EPEC Map promoter was introduced into C. rodentium, Map expression persisted up to $17 \mathrm{~d}$, indicating that Map expression is differentially regulated in EPEC and C. rodentium. In summary, our data show that Map expression is distinctively regulated from other effectors in EPEC, but not C. rodentium, indicating its importance in the initial phase of EPEC infection.

\section{Tu2015}

Antagonistic Effects of Probiotic E. Coli Nissle 1917 on Various EHEC Strains Stefan A. Rund, Tobias A. Oelschlaeger

Introduction Due to the centennial application and extensive studies of its efficiency and molecular biology, E. coli Nissle $1917(\mathrm{EcN})$ is among the best characterized probiotics. $\mathrm{EcN}$ is approved as a pharmaceutical in the EU and widely used in treatment of gastrointestinal disorders. In 2011, pathogenic Shiga toxin-producing E. coli O104:H4 caused the largest outbreak of enterohemorrhagic E. coli (EHEC) recorded so far. In this study, the influence of the probiotic E. coli Nissle strain 1917 on adhesion and growth of pathogenic E. coli strains (pEc) was investigated in vitro. We used EHEC (O157:H7), enteroaggregative E. coli (EAEC, O104:H4), uropathogenic E. coli (UPEC, O153:H31), and two O104:H4 EHEC (clinical isolates) from the recent outbreak in Germany in our experiments. Shiga toxin production of the EHEC strains was elucidated in co-culture with $\mathrm{EcN}$. We are currently investigating the mechanisms responsible for these important antagonistic effects of EcN on pEc strains. Methods 24-well-plates were coated with the human gut epithelial cell lines Caco2 or LS174T, to determine the adhesion of living bacteria to those cells. Single cultures of $\mathrm{EcN}, \mathrm{pEc}$, and co-cultures at ratios of $1: 1$ and 10:1 (EcN:pEc) were incubated for $2 \mathrm{~h}$ with the epithelial cells, followed by washing, epithelial cell lysis and plating of serial dilutions of the resulting bacterial suspension on agar plates. Furthermore, the growth of the bacteria in each well was determined at $t=0 h, t=2 h, t=5 h$ and $t=24 h$ by plating of serial dilutions on agar plates. Shiga toxin production of single cultures of EcN, EHEC, and co-cultures at ratios of 1:1 and 10:1 (EcN:EHEC) was analyzed after 24h incubation via Verotoxin ELISA. Results EcN significantly reduced the adhesion of all tested pEc strains to Caco2 and LS174T cells by up to $99 \%$ for EAEC O104:H4, UPEC O153:H31, EHEC O157:H7 and to >80 $\%$ for O104:H4 EHEC, in a co-culture with 10-fold EcN. In addition, inhibition of growth, due to killing of pathogenic E. coli, occurred. The number of EHEC (O157:H7) in coculture with EcN, for example, dropped to a 100-fold lower cell count than the number of EcN after 24 h. Shiga toxin production of EHEC O104:H4 (TY3730) and EHEC EDL933 in co-culture with EcN in DMEM medium at a 10:1 ratio (EcN:EHEC) decreased by 80\%. Microcins were identified as the first factor contributing to the antagonistic effects of EcN on $\mathrm{pEc}$ strains. Discussion In our experiments reduction of Shiga toxin production and adhesion to human gut epithelial cell lines, as well as inhibition of EHEC growth was observed. This is a good indication that $\mathrm{EcN}$ might be effective in preventing an infection with EHEC strains and even assist in the treatment of acutely affected patients. However, more experiments and clinical trials have to be conducted before EcN can be considered as a treatment option for EHEC infections.

\section{Tu2016}

Antimicrobial Resistance of Shigella Isolates in Alanya, Turkey Askin Erdogan, Haluk Erdogan

Aim: The increasing rate of resistance among enteric pathogens has recently limited antibiotic therapy. The aim of this study is to evaluate the antimicrobial activity of antimicrobial agents against Shigella isolates in Alanya, an important tourism center in Turkey. Materials and Methods:The study was carried out between 1 January 2004 and 1 September 2011. Shigella spp isolated from stool samples at Baskent University Alanya Research and Medical Center were stored at $-80 \mathrm{C}$. Faecal samples were cultured onto Eosine Methylene Blue (EMB) agar and Hektoen Enteric agar for the isolation of Shigella spp., which were identified biochemically by the standard methods and grouped serologically by slide agglutination with specific antisera (Denka Saiken, Tokyo, Japan). Antimicrobial susceptibility was determined by the Kirby-Bauer disk diffusion method following the Clinical and Laboratory Standards Institute (CLSI) guidelines. The antibiotics tested against Shigella spp. were ampicillin, trimethoprimsulphamethoxazole, tetracycline, chloramphenicol, ciprofloxacine and ceftriaxone. Quality control was ensured by testing Esherichia coli ATCC 25922. In this study, multidrug resistance (MDR) is defined as the presence of resistance to at least two antimicrobial agents. Results:A total of 26 Shigella isolates (12 were S. sonnei, 8 S. flexneri, 4 S.dysenteriae and $2 \mathrm{~S}$. boydii) were assessed for their antimicrobial susceptibility patterns. These strains showed high levels of resistance to trimethoprim-sulphamethoxazole (58\%), ampicillin (54\%), and tetracycline (50\%). Chloramphenicol resistance were found to be $12 \%$. Ceftriaxone resintance was found to be $7.5 \%$. No isolate was resistant to ciprofloxacine. Forty-six percent were multidrug resistant. Conclusions: Shigella sonnei and Shigella flexneri are the predominant pathogenic species responsible for shigellosis in our study. The prevalence of antimicrobia resistance in Shigella isolates to the most commonly used antibiotic such as ampicillin, tetracycline, trimethoprim-sulphamethoxazole is high. Ceftriaxone resistance is worrying. Quinolone resistance was not found.

\section{Tu2017}

Gut Microbiota Urease Activity Regulates Serum Urea Levels on a Low Protein Diet

Evelyn K. Hsu, Ying-Yu Chen, Evgueni I. Daikhin, Oksana Horyn, Ilana Nissim, Christian Hoffmann, Kyle Bittinger, Frederic D. Bushman, Marc Yudkoff, Gary D. Wu

Background: The gut microbiota is one of the most densely populated bacterial communities on Earth and plays an important role in the metabolic homeostasis of the mammalian host. Classic studies in physiology have shown that it helps to maintain host nitrogen balance through the hydrolysis of colonic urea and the subsequent absorption of resultant ammonia Reduction in the consumption of protein due to illness, as part of medical therapy, or as a result of global societal factors, can have profound effects on host metabolism. In this study, we examined the role of the gut microbiota on metabolic disturbances associated with the consumption of a low protein diet (LPD). Methods and Results: Female C57B/6J mice were fed either a normal chow $(20 \% \mathrm{kcal}$ protein) or LPD ( $3 \% \mathrm{kcal}$ protein) for 1 month. Metabolic phenotyping revealed that LPD mice failed to gain weight and suffered from a profound defect of water conservation manifested as severe polydypsia and polyuria. This was due to the inhibition of hepatic ureagenesis and the reduction in serum urea levels. $16 \mathrm{~S}$ rRNA gene sequencing revealed that the gut microbiota was altered significantly by a LPD but in a manner that was not directly associated with this metabolic phenotype. Remarkably, the administration of oral antibiotics to LPD mice restored serum urea levels and prevented the development of polyuria and polydypsia. Antibiotic-induced restoration of serum urea levels did not alter host ureagenesis in LPD mice since hepatic urea cycle gene expression remained suppressed. By contrast, ${ }^{13} \mathrm{C}$-urea breath test and direct measurements of fecal urease activity revealed that oral antibiotic treatment dramatically reduced colonic urea hydrolysis thereby helping to preserve circulating host urea levels. Interestingly, the ${ }^{13} \mathrm{C}$-urea breath test also showed that colonic urease activity was reduced by a LPD demonstrating the impact of diet on gut microbiota function. Conclusions: Our results demonstrate that oral antibiotics alter the host-microbial urea equilibrium in mice fed a LPD by reducing the capacity of the gut microbiota to hydrolyze host-produced urea. When ureagenesis is suppressed by a LPD, serum levels of urea fall as the gut microbiota continues to consume urea in the colon. Antibiotic treatment of LPD mice restores serum urea levels by reducing the hydrolysis of urea in the colon thereby disrupting the diffusion gradient and reducing the movement of urea into the colon. Finally, alterations in gut microbiota composition as well as the reduction of its urease activity on a LPD indicate that diet alters not only the structure but also the function of the gut microbiota. This model system may prove to be very valuable in assessing interventions that modulate gut microbiota urease activity for the treatment of disease.

\section{Tu2018}

Maternal Antibiotic Treatment Peripartum Has Differential Consequences on Protein Expression of Intestinal Cytoprotective Heat Shock Proteins in Her Offspring

Marie-Edith Arnal, Jean-Paul Lalles

Background: Peripartum antibiotics are thought to have negative consequences on intestinal barrier function, immune system development and long-term health. These effects might be related to disturbances in neonatal bacterial colonization. Protective heat shock proteins 25 and 72 are induced physiologically by luminal bacteria in cultured intestinal epithelial cell (IEC) and in mouse intestine. HSP60 has been recently shown to contribute to IEC protection in vitro but data in vivo are lacking. We aimed at testing the hypothesis that early alterations in gut microbial colonization impact intestinal cytoprotective HSPs. Methods: Sows received amoxicillin orally $(40 \mathrm{mg} / \mathrm{kgBW} / \mathrm{d}, \mathrm{ATBQ} \mathrm{n}=1 \mathrm{l}$; vs. untreated CTL, $\mathrm{n}=12$ ) 
around parturition (d-10 till $\mathrm{d}+21)$. Offspring (1/litter) were sacrificed during the suckling period (d14, d28) and after weaning (d42), and ileal tissues were collected. HSP27, HSP60, HSP70 and cognate HSC70, as well as the transcription factor HSFl were determined by western blotting and tissue levels expressed relative to $\beta$-actin. Enzyme activity of intestinal alkaline phosphatase (IAP), an HSP-like protein crucial in the detoxification of pro-inflammatory bacterial components (e.g. LPS) was also investigated in comparison. Results: Ileal tissue levels of HSP27 in offspring were unaffected by antibiotic treatment of mothers. HSP60 tended to be $(\mathrm{P}=0.07)$ and $\mathrm{HSC} 70$ was $(\mathrm{P}<0.05)$ higher in offspring born to ATBQ sows than in CTL (+40 and $+24 \%$, respectively). By contrast, HSP70 was much lower in offspring born to ATBQ sows than in CTL $(\mathrm{P}<0.01)$ and this difference was significant $(\mathrm{P}<0.01)$ at day $28(-69 \%)$ and day $42(-59 \%)$ (treatment by age interaction, $\mathrm{P}<0.05)$. Ileal protein expression of HSFl in offspring was unaffected by ATBQ treatment of sows or offspring post-natal age. In comparison to HSPs, IAP activity displayed a treatment by time interaction: it was twice lower at day 14 in offspring born to ATBQ sows than in CTL $(P<0.01)$ with no differences at days 28 and 42 . Conclusion: Maternal antibiotic treatment peripartum had differential consequences on protein expression of intestinal heat shock proteins in her offspring in the swine. This did not involve the modulation of HSP-gene transcription factor HSFl, suggesting alternate regulatory mechanisms. Collectively, the data suggest complex, time-dependent interactions between colonizing maternal fecal microbiota and offspring intestinal HSP expression. Changes in offspring intestinal microbiota and long-term effects of perinatal antibiotic treatment on offspring intestinal HSP family are being investigated.

\section{Tu2019}

Neonatal Probiotic Administration Has Long-Lasting Effects on Gut Permeability Responses to Stress in Adult Pigs Born to Sows Treated With Antibiotics Around Parturition

Jean-Paul Lalles, Dominique Bertacchini, Gerard Savary, Hauke Smidt

Background: Peripartum antibiotics are thought to have long-lasting negative consequences on intestinal barrier function, immune system development and long-term health, e.g. allergy. Various kinds of stress (e.g. nutrition, environment) can be deleterious to gut barrier function. We have shown that broad spectrum antibiotic administration to sows around parturition alters various facets of gut barrier in their offspring during development and in adulthood. We tested with this model the hypothesis that early administration of a probiotic to offspring can modulate gut barrier function in young adults depending on diet and stressor. Methods: Sows $(n=20)$ received amoxicillin orally $(40 \mathrm{mg} / \mathrm{kgBW} / \mathrm{d}$ ) around parturition (day-10 to day+21) in order to induce gut barrier disturbances in offspring. Piglets from half of the litters received orally a probiotic suspension (Lactobacillus sobrius, 108/ml, $1 \mathrm{ml} /$ pig and intervention, PROB) at birth and then three times a week until postnatal day PND21. Offspring were reared under the sow until weaning at PND28. They were reared with a similar diet until PND141. Then, each group was split into two halves. One half in each group remained on the control (low fat, LF 2\%) diet while the other halves were fed a high fat (HF, 11\%) diet. Pigs were sacrificed at PNDl69 and pieces of ileum and colon were collected and mounted in Ussing chambers (UC). UC treatment included control, oxidative stress (monochloramine) and mast cell degranulation stress (48/80). Mucosal para- and trans-cellular permeabilities (PCP, TCP) were measured using FD4 and HRP, respectively. Results: Early PROB supplementation had no effect on offspring basal ileal and colonic permeabilities. Ileum from PROB pigs fed LF diet displayed reduced PCP in oxidative $(\mathrm{P}<0.05)$ and degranulation $(\mathrm{P}<0.10)$ conditions. No differences between PROB and CTL were seen for colonic PCP. Contrasting with this, ileal TCP was much higher ( $\mathrm{x} 2$ to 3 ) in PROB pigs with both LF and HF diets $(\mathrm{P}<0.01)$ after degranulation stress. An interaction $(\mathrm{P}<0.10)$ between early PROB treatment and adult diet was observed for TCP after oxidative stress (decrease with LF, increase with HF). Colonic TCP was increased after both oxidative and degranulation stress in PROB pigs fed LF (but not HF) diet $(\mathrm{P}<0.05)$. Conclusion: Our data in the swine model suggest that probiotics provided early to gut barrier-disturbed offspring born to mothers treated with broad-spectrum antibiotic around parturition can contribute to long-term modulation of gut barrier function following stress. However, the responses are complex and depend on gut site, and diet composition and type of stressor in adluthood. Work is in progress to investigate long-term changes in gut microbiota composition induced by early probiotic administration.

\section{Tu2020}

Chronic Ingestion of Lactose: Malabsorber Host and Intestinal Microbiota Adaptations

Virginie Alexandre, Annaig Lan, François Blachier, Robert Benamouzig, Yolanda Sanz, Daniel Tomé, Anne-Marie Davila

Introduction: Physiological and metabolic consequences of chronic lactose ingestion by malabsorbers are poorly described. In order to highlight the suspected modulations and adaptations, intestinal microbiota and host's physiology have been in vivo studied in hypolactasic rats, over a prolonged ingestion of lactose. Materials and methods: Two groups of Wistar male rats ingested a diet containing $25 \%$ of lactose (L25 group) or $25 \%$ of sucrose (pair-fed S25 group). Consecutive slaughters were performed over 6 days of the diet. Bacterial groups' evolutions (qPCR), short-chain-fatty-acids (SCFA) concentrations and $\beta$ galactosidase activity were evaluated in caecal and colonic contents. Concomitantly, SCFA were quantified in portal blood, myeloperoxydase activity (MPO) was evaluated in colonic mucosa and histological samples were analysed. Results: L25 vs S25 comparisons over time reveal a stability of the total counts of bacteria, despite proliferations of fermentative groups with or without $\beta$ galactosidase activity, with a segment-dependent dynamic. In the L25 group, Bacteroides predominate in caecum and distal colon (diet effect $\mathrm{p}<10-4$ ). In proximal colon, groups' evolutions are noticed but stimulate at day 6: Bacteroides, enterobacteria, lactobacilli (diet effect $\mathrm{p}<10-4)$. Sulfate-reducing bacteria decrease in all segments in favour of acetogenic bacteria in caecum and proximal colon, and also archeae methanogens in distal colon (diet effect $\mathrm{p}<10-4$ ). Consecutively, $\beta$ galactosidase activity is higher in caecum of L25 group (time*diet effect $\mathrm{p}<0.05$ ). Moreover, SCFA colonic concentrations become significantly higher in L25 group, with a time* diet effect in caecum $(\mathrm{p}<0.05)$ and distal colon $(\mathrm{p}=0.002)$. SCFA blood concentrations significantly increase at day 1 , then stabilise at day 2 . At last,
MPO activity and mucosa histology underline a transitional inflammation at days 1 and 2 in the L25 group, especially with an MPO activity higher in proximal colon $(\mathrm{p}=0.04)$ and distal colon ( $\mathrm{p}=0.03)$, a smaller ratio (mucosa area/ section area) $(\mathrm{p}=0.03)$ and a higher ratio (goblet cells/ colonocytes) ( $\mathrm{p}=0.04)$. Conclusions: Prolonged lactose ingestion modifies both composition and activity of the intestinal microbiota, stimulating a turn-over of the lactose colonic metabolites and a reduction of the mucosa inflammation. These findings underline the roles of microbiota-host interplays and suggest that small but regular amounts of lactose could improve the malabsorber host's tolerance.

\section{$\mathrm{Tu} 2021$}

Intestinal Epithelial Cells Apically Secrete Exosomes Taken up by Neighboring Epithelial Cells and Bacteria

Poonam Rakhya, Bo Xiao, Hamed Laroui, Didier Merlin

Background and Aims: Exosomes are small membrane vesicles of endosomal origin secreted from a variety of cell types. Exosomes contain proteins, mRNAs, and microRNAs, and may play roles as mediators of cell-to-cell communication. Herein, we characterized exosomes secreted by intestinal epithelial cells and investigated exosome uptake by epithelial cells and bacteria. Methods: Release of exosomes from Caco2-BBE monolayers grown under basal or inflammatory conditions was evaluated by differential ultracentrifugation after growth of such cells in conditioned apical and basolateral media. Exosome size and morphology were assessed employing light-scattering techniques, atomic force microscopy, and scanning electron microscopy. The exosomal marker CD63 was used to assess the purity of exosome preparations. Fluorescent microscopy was employed to visualize intracellular uptake of secreted Caco2-BBE exosomes (fluorescently labeled by electroporation of FITC-siRNA complexed with PEI) by macrophages RAW264.7, Caco2-BBE monolayers, and bacteria. Results: Caco2-BBE cell monolayers released vesicles $80-150 \mathrm{~nm}$ in diameter bearing the exosomal marker CD63 from the apical but not from the basolateral side, and the extent of such release was increased when the monolayers were pre-stimulated with LPS $(10 \mathrm{ng} / \mathrm{mL}$ for 4h). FITC-labeled secreted exosomes from Caco2-BBE monolayers were taken up by such monolayers (70\%), macrophages (30\%), and bacteria. Conclusion: Caco2-BBE cell monolayers specifically secrete exosomes toward the luminal side of the intestinal cell monolayer. Such apical exosome secretion by intestinal epithelial cells is a regulated process. Secreted exosomes may fuse with neighboring epithelial cells, thus transferring membranous and cytoplasmic contents from one cell to another, and may also be taken up by bacteria of the colonic lumen. Secreted exosomes may play key roles in epithelial cell/cell and epithelial cell/bacterium communication under pathophysiological conditions.

\section{Tu2022}

Role of Food and Enterobacteria in the Formation and Prevention of Small Intestinal Lesions Induced by Enteric Coated Aspirin in Cats Hiroshi Satoh, Kikuko Amagase, Ami Yokoi, Taiki Kawabata, Koji Takeuchi

BACKGROUND/AIM: Recently, low-dose aspirin (ASA) has been widely used as the firstchoice drug for the prophylaxis and treatment of thrombosis. Enteric-coated (EC) ASA has been used to limit gastric lesions as a side effect, but even low doses of EC ASA often cause mucosal damage in the small intestine in humans. However, there are few studies on the role of food and enterobacteria in the formation and prevention of the intestinal damage. We examined the effect of various foods and antibiotic on the formation of intestinal lesions induced by EC-ASA in cats. METHODS: Adult cats were used (4 to 6 animals per group). Several types of diet containing dietary fiber (DF) at various percentages were given to the animals twice daily during the experiment. In the fed group, 1 EC ASA tablet (containing $100 \mathrm{mg}$ ASA) was administered p.o. once daily after the morning meal for 7 days, whereas in the fasted group, EC ASA was given in the morning after an overnight fast. The animals were sacrificed $24 \mathrm{~h}$ after the final EC-ASA dose and mucosal lesions in the GI tract were examined. RESULTS: 1) EC ASA tablet did not cause any visible lesions in the stomach. In the fasted condition, EC ASA given orally once a day for 7 days caused mild lesions in the duodenum and small intestine; the mean lesion area (MLA) was $0.17 \pm 0.07 \mathrm{~cm} 2$ and $0.23 \pm 0.07 \mathrm{~cm} 2$, respectively. When EC ASA was given after feeding of regular dry food (Dry) containing $2.8 \% \mathrm{DF}$, the duodenal and intestinal lesions were markedly increased; the MLA was $0.95 \pm 0.11 \mathrm{~cm} 2$ and $1.11+0.31 \mathrm{~cm} 2$ ( $\mathrm{P}<0.01$ and $0.05 \mathrm{vs}$, fasted), respectively. 2) In the cats ate canned food (Can) containing $0.4 \% \mathrm{DF}$, the lesions were markedly decreased; the MLA was $0.01 \pm 0.01 \mathrm{~cm} 2$ and $0.08 \pm 0.05 \mathrm{~cm} 2(\mathrm{P}<0.001$ and 0.01 vs. Dry), respectively. The addition of insoluble DF (cellulose $6 \%$ ) to the canned food appeared the lesions again; the MLA was $0.32 \pm 0.20 \mathrm{~cm} 2$ and $0.69 \pm 0.32 \mathrm{~cm} 2$ ( $P<0.05$ vs. Can), respectively. The addition of soluble DF (pectin 6\%) to dry food markedly decreased the lesions; the MLA was $0.07 \pm 0.07 \mathrm{~cm} 2$ and $0.10 \pm 0.03 \mathrm{~cm} 2$ ( $P<0.001$ and 0.01 vs. Dry). 3) Neomycin (20 mg/kg, p.o.) given $30 \mathrm{~min}$ before morning meal (Dry) for 7 days decreased the lesions in the small intestine, the MLA was $0.21 \pm 0.03 \mathrm{~cm} 2$ ( $\mathrm{P}<0.05$ vs. Dry). CONCLUSIONS: 1) Adverse effects of EC ASA on the duodenal and intestinal mucosa depend on the feeding conditions (fasted or fed) when EC ASA was administered, i.e., the lesions were markedly increased when EC ASA was given after feeding of dry food. 2) Contents of diet, especially insoluble DF such as cellulose and soluble DF such as pectin, play an important role in the formation and prevention of duodenal and intestinal lesions induced by EC ASA. 3) Gram negative enterobacteria such as E. coli may play some role in the formation and aggravation of intestinal lesions. 